\title{
Características físico-químicas e composição centesimal de queijos de coalho comercializados em cidades do Ceará
}

Physico-chemical characteristics and centesimal composition of rennet cheeses marketed in cities of Ceará

Características fisicoquímicas y composición centesimal de los quesos de cuajo comercializados en las ciudades de Ceará

Edilene Ferreira da Silva

ORCID: https://orcid.org/0000-0001-7073-7962

Universidade Federal da Santa Catarina, Brasil E-mail: silvaedilene16@hotmail.com

Priscila Luana da Silva

ORCID: https://orcid.org/0000-0002-1655-7394 Instituto Federal de Educação, Ciência e Tecnologia do Ceará, Brasil E-mail: priscilaluana.tecalimentos@gmail.com Samuel Carneiro de Barcelos

ORCID: https://orcid.org/0000-0002-1706-9114 Universidade Federal do Ceará, Brasil E-mail: samuelbarcelos05@gmail.com

Vera Lúcia Viana do Nascimento

ORCID: https://orcid.org/0000-0003-0071-3863 Instituto Federal de Educação, Ciência e Tecnologia do Piauí, Brasil E-mail: veravnascimento@gmail.com

Lidiana de Siqueira Nunes Ramos ORCID: https://orcid.org/0000-0003-3016-4412 Instituto Federal de Educação, Ciência e Tecnologia do Piauí, Brasil E-mail: lidiana@ifpi.edu.br

Antônio Belfort Dantas Cavalcante $\nmid 31.05 .2020$

ORCID: https://orcid.org/0000-0001-8440-2979 Instituto Federal de Educação, Ciência e Tecnologia do Ceará, Brasil E-mail: belfort.dantas@ifce.edu.br

\begin{abstract}
Resumo
Queijo de coalho é um produto popular da cultura nordestina, sendo comum o processo de elaboração com uso de leite cru, o conhecimento prévio da composição físico-química e centesimal do alimento é relevante, tanto por influenciar na escolha saudável, como para obter dados a respeito do que está sendo consumido com qualidade. Objetivou avaliar as características físico-químicas e a composição centesimal do queijo de coalho comercializado em cidades do Ceará. Foram coletadas cinco diferentes marcas de queijos de coalho (A, B, C, D e E) em três repetições, encaminhadas para a Planta Piloto de Leite e Derivados do IFCE - Campus Limoeiro do Norte (CE). As amostras foram homogeneizadas em ralador doméstico, e acondicionadas sob refrigeração $\left(4 \pm 2{ }^{\circ} \mathrm{C}\right)$ até o momento das análises. Análises realizadas: físico-químicas $\left(\mathrm{pH}\right.$, atividade de água $\left(\mathrm{A}_{\mathrm{w}}\right)$, acidez titulável e cor), composição centesimal (umidade, proteínas, cinzas, lipídios, carboidratos totais, e valor calórico total). Os dados estatísticos por delineamento inteiramente casualizados, foram submetidos à análise de variância (ANOVA) e teste de Tukey, através do programa R Core Team, 2018, versão 3.5.0, nível de 5\% de significância. As análises físico-químicas dos queijos de coalho demonstraram variabilidade entre as amostras, associadas às condições da produção, variação da temperatura de armazenamento e comercialização dos queijos. A composição centesimal e o valor calórico expresso em Kcal variaram entre as amostras estudadas, estando os teores de umidades de acordo com o que a legislação preconiza para queijo de coalho e os valores lipídicos abaixo do mínimo exigido pela legislação.
\end{abstract}

Palavras-chave: Conservação; Leite; Produto; Processamento; Qualidade.

\begin{abstract}
Coalho cheese is a popular product of the Northeastern culture, and the process of making it using raw milk is common, prior knowledge of the physical-chemical and centesimal composition of the food is relevant, both for influencing the healthy choice and for obtaining data from regarding what is being consumed with quality. The objective was to evaluate the physical-chemical characteristics and the chemical composition of rennet cheese
\end{abstract}


marketed in cities in Ceará. Five different brands of rennet cheese (A, B, C, D and E) were collected in three replications, sent to the Pilot Plant for Milk and Derivatives of IFCE - Campus Limoeiro do Norte (CE). The samples were homogenized on a household grater, and stored under refrigeration $\left(4 \pm 2{ }^{\circ} \mathrm{C}\right)$ until the time of analysis. Analyzes performed: physical-chemical $(\mathrm{pH}$, water activity (Aw), titratable acidity and color), proximate composition (moisture, proteins, ash, lipids, total carbohydrates, and total caloric value). The statistical data by completely randomized design were subjected to analysis of variance (ANOVA) and Tukey's test, through the program R Core Team, 2018, version 3.5.0, level of significance of 5\%. Physical-chemical analyzes of rennet cheeses showed variability between samples, associated with production conditions, variation in the temperature of storage and commercialization of cheeses. The chemical composition and the caloric value expressed in Kcal varied between the samples studied, with the moisture content in accordance with what the legislation recommends for rennet cheese and the lipid values below the minimum required by the legislation.

Keywords: Conservation; Milk; Product; Processing; Quality.

\section{Resumen}

El queso Coalho es un producto popular de la cultura nororiental, y el proceso de elaboración con leche cruda es común, el conocimiento previo de la composición físico-química y centesimal del alimento es relevante, tanto para influir en la elección saludable como para obtener datos de respecto a lo que se consume con calidad. El objetivo fue evaluar las características físico-químicas y la composición química del queso cuajo comercializado en las ciudades de Ceará. Se recolectaron cinco marcas diferentes de queso cuajo (A, B, C, D y E) en tres réplicas, enviadas a la Planta Piloto de Leche y Derivados de IFCE - Campus Limoeiro do Norte (CE). Las muestras se homogeneizaron en un rallador doméstico y se almacenaron en refrigeración $\left(4 \pm 2{ }^{\circ} \mathrm{C}\right)$ hasta el momento del análisis. Análisis realizados: físico-químico ( $\mathrm{pH}$, actividad hídrica (Aw), acidez titulable y color), composición próxima (humedad, proteínas, cenizas, lípidos, carbohidratos totales y valor calórico total). Los datos estadísticos mediante diseño completamente aleatorizado fueron sometidos a análisis de varianza (ANOVA) y prueba de Tukey, mediante el programa $\mathrm{R}$ Core Team, 2018, versión 3.5.0, nivel de significancia del 5\%. Los análisis físico-químicos de los quesos de cuajo mostraron variabilidad entre muestras, asociada a las condiciones de producción, variación en la temperatura de almacenamiento y comercialización de los quesos. La composición química y el valor calórico expresado en Kcal varió entre las muestras estudiadas, siendo el contenido de humedad acorde a lo recomendado por la legislación para el queso cuajo y los valores lipídicos por debajo del mínimo exigido por la legislación.

Palabras clave: Conservación; Leche; Productos; Procesando; Calidad.

\section{Introdução}

O leite é um alimento altamente consumido e nutritivo amplamente consumido pela população em geral. Sendo um produto cru, é submetido ao processamento térmico para destruição de bactérias patogênicas, redução de microrganismos deteriorantes e inativação de enzimas endógenas desfavoráveis, de modo a garantir a segurança dos consumidores de leite e derivados, além de garantir a segurança do leite desde o momento da ordenha até o consumidor final, buscando prevenir qualquer tipo de contaminação (Liao et al., 2019; Rosseto, Batistella \& Veiga 2020). Além do leite e seus derivados serem bastante consumidos devido às propriedades sensoriais, é necessário destacar o importante papel do leite na nutrição humana ao longo da história da humanidade (García-Gómez et al., 2019).

A fabricação de queijo há milhares de anos, envolve basicamente a conversão do leite líquido em coalhada sólida, seguida ou não da evolução bioquímica da coalhada em queijo curado (Reis \& Malcata, 2011). Entende-se por queijo de coalho, o queijo que se obtém por coagulação do leite por meio do coalho ou outras enzimas coagulantes apropriadas, complementada ou não pela ação de bactérias lácteas selecionadas e comercializado normalmente com até 10 (dez) dias de fabricação, esse queijo tem como característica alta umidade, massa semicozida ou cozida e apresentando um teor de gordura nos sólidos totais variável entre 35,0 e 60,0\% (Brasil, 2001). Esse mesmo Regulamento Técnico de Identidade e Qualidade de Queijo de Coalho (Brasil, 2001) estabelece os requisitos mínimos de qualidade que deverá cumprir o queijo de coalho destinado ao consumo humano e descreve as características de como deve ser o mesmo e sua definição.

Segundo a legislação os requisitos físico-químicos correspondem às características de composição e qualidade dos queijos de média a alta umidade, conforme estabelecido no Regulamento Técnico de Identidade e Qualidade de Queijos (Brasil, 1996), estão também fixas a identidade e os requisitos mínimos de qualidade que deverão possuir os queijos, 
abordando os requisitos de teor de gordura, conteúdo de umidade e padrões microscópicos como físico-químicos. Além dessas análises, a avaliação da composição centesimal também é um método de importância para se avaliar a qualidade dos queijos, visto que a qualidade não é definida por um único parâmetro, mas por um conjunto que forma o produto final.

A coagulação enzimática do leite é uma etapa importante no processo de fabricação de queijos, no caso do queijo de coalho é obtida através da adição do coalho, que é uma mistura de enzimas proteolíticas e lipolíticas, como a quimosina, pepsina e lipase, onde sua atividade no leite favorece a degradação das proteínas caseínas através da interrupção das ligações peptídicas covalentes da $\kappa$-caseína formada entre fenilalanina e metionina (Selin et al., 2018).

A importância nutricional do queijo foi confirmada em um estudo recente, onde foi observado que durante a fermentação do leite, a concentração de fosfolipídios sofre um grande aumento, aumentando o valor nutricional desse derivado lácteo (Ferreiro \& Rodríguez-Otero, 2018). Além disso, estudos recentes sugerem que compostos bioativos como lipídios funcionais (CLA), vitaminas, peptídeos antimicrobianos, ácido $\gamma$-aminobutírico e exopolissacarídeos com potencial efeito na prevenção de doenças pode ser liberados por algumas bactérias lácticas (Santiago-López et al., 2018).

As análises físico-químicas em alimentos têm o objetivo de avaliar se as amostras estão de acordo com os Padrões de Identidade e Qualidade - PIQ, pesquisar fraudes, adulterações, alterações físico-químicas, organolépticas, e outras características que possam afetar a qualidade do produto. Derivados lácteos precisam passar pela avaliação de sua qualidade, sendo que no caso do queijo de coalho, por suas peculiaridades regionais no Brasil, tal avaliação se torna ainda mais importante para garantir um produto de qualidade assegurada ao consumidor.

O conhecimento prévio da composição do alimento é relevante, tanto por influenciar na escolha, como também para obter dados sobre qualidade dos produtos que estão sendo consumidos, já que atualmente a população tem mostrado uma preocupação com vida saudável e com alimentos que influenciam diretamente na qualidade dos mesmos. Diante disso, objetivou avaliar as características físico-químicas e de composição centesimal do queijo de coalho comercializado em cidades do Ceará.

\section{Metodologia}

A seguinte pesquisa é de natureza quantitativa e qualitativa, pois analisou-se as diferentes amostras coletadas e obteve-se dados a respeito das mesmas, que são suportes para avaliar sua qualidade. Muitas vezes os métodos qualitativos podem se transformar em quantitativos, ou um complementar o outro por meio do emprego de questões fechadas, desde que estes influenciam diretamente na interpretação dos dados obtidos no estudo (Pereira et al., 2018). A pesquisa foi realizada em três etapas diferentes, como descrito a seguir:

\section{Coleta de amostras e preparo para análises}

Foram coletadas 5 amostras de diferentes marcas de queijo de coalho, no período de março a abril de 2016, cada amostra de uma marca diferente e comercializada em cidades do Ceará. Cada amostra coletada pesava 500 gramas, em embalagens apropriadas (embalagem original). As amostras foram codificadas como: Queijo tipo de Coalho (QtC), A, B, C, D e E. As amostras adquiridas foram acondicionadas em caixa térmica e imediatamente encaminhadas à Planta Piloto de Leite e Derivados do Instituto Federal de Educação, Ciência e Tecnologia do Ceará, Campus Limoeiro do Norte, Ceará, Brasil e, armazenadas sob temperatura de refrigeração $\left(4 \pm 2{ }^{\circ} \mathrm{C}\right)$ até o início do processamento das amostras.

As amostras de QtC foram processadas em ralador de queijo caseiro para redução das amostras, em seguida as amostras foram acondicionadas em embalagens plásticas e colocadas sob refrigeração $\left(4 \pm 2{ }^{\circ} \mathrm{C}\right)$, até o momento das análises. 


\section{Análises físico-químicas e composição centesimal}

As análises físico-químicas realizadas nos QtC foram: $\mathrm{pH}$ em potenciômetro de bancada (Lucadema, Luca-210), atividade de água $\left(\mathrm{A}_{\mathrm{w}}\right)$ a $25{ }^{\circ} \mathrm{C}$ em medidor de atividade de água (AquaLab-BrasEq), acidez em mg de ácido lático.g ${ }^{-1}$, realizadas em triplicatas, conforme metodologia preconizada pelo Instituto Adolfo Lutz (2008). A cor foi determinada de acordo com o sistema de cores $L^{*}, a^{*}, b^{*}$ da International Commission on Illumination (CIE), utilizado um colorímetro digital MiniScan EZ, HunterLab (BrasEq, Jarinu-São Paulo) e a leitura feita diretamente sobre a superfície da amostra (nas amostras de QtC integras, antes de serem homogeneizadas) em triplicata. A partir da emissão de um feixe de luz da lente do colorímetro, medido por reflectância e, os resultados foram expressos pelo equipamento na escala de cores da Hunter Lab, que incluem as variáveis $L^{*}, a^{*}, b^{*}$, onde, $L^{*}$ (luminosidade), $a^{*}$ (verde a vermelho) e $b^{*}$ (Azul a amarelo).

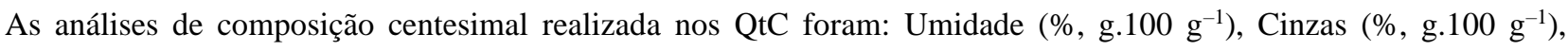
Lipídios (\%, g.100 g ${ }^{-1}$ ) e Proteínas (\%, g.100 g ${ }^{-1}$ ), usando fator de conversão de 6,38, conforme o Instituto Adolfo Lutz (2008). Os carboidratos totais (\%, g.100 g-1), por diferença e Valor Calórico Total (VCT) em kcal.100 g g $^{-1}$ calculado pela soma dos resultados das multiplicações dos valores encontrados de proteína, carboidratos, lipídios pelos seus respectivos fatores de conversão (4, 4 e 9 kcal) (Atwater, 1910). Todas as análises foram realizadas em triplicatas.

\section{Análise estatística}

Foram verificados os pressupostos de normalidade e homogeneidade das variáveis, respectivamente pelo teste de Shapiro-Wilk e Bartlett. Os dados obtidos foram submetidos à Análise de Variância e teste de Tukey $(p<0,05)$ para comparação das médias. O software estatístico utilizado para as análises foi o programa estatístico R Core Team, 2018, versão 3.5.0 (R Core Team, 2018).

\section{Resultados e Discussão}

A Tabela 1 apresenta os valores de $\mathrm{pH}$, acidez titulável em mg de ácido lático.g ${ }^{-1}$ e atividade de água $\left(\mathrm{A}_{\mathrm{w}}\right)$ nos Queijos de Coalho (QtC) comercializados em cidades do Ceará. De maneira geral, observou-se diferença significativa $(p<0,05)$ entre todas as amostras de QtC para todos os valores de $\mathrm{pH}$ e acidez titulável, exceto para $\mathrm{pH}$ entre as amostras QtC-C e D e, para acidez, entre as amostras QtC-C e E, que apresentaram semelhança significativa entre si $(p>0,05)$.

Tabela 1. Análises físico-químicas [média $(\mathrm{CV} \%)]^{1}$ dos queijos de coalho comercializados em cidades do Ceará.

\begin{tabular}{|c|c|c|c|}
\hline \multirow[b]{2}{*}{ Marcas de QtC* } & \multicolumn{3}{|c|}{ Parâmetros Físico-químicos } \\
\hline & $\mathbf{p H}$ & $\begin{array}{c}\text { Acidez titulável } \\
\left.\text { (mg de ác.lático.g }{ }^{-1}\right)\end{array}$ & Atividade de água $\left(\mathrm{A}_{\mathrm{w}}\right)$ \\
\hline QtC-A & $5,30^{\mathrm{d}}(0,58)$ & $0,28^{\mathrm{a}}(1,89)$ & $0,697^{\mathrm{b}}(0,63)$ \\
\hline QtC-B & $6,29^{\mathrm{a}}(0,66)$ & $0,10^{\mathrm{b}}(5,61)$ & $0,710^{a}(1,15)$ \\
\hline QtC-C & $6,13^{\mathrm{b}}(0,41)$ & $0,08^{\mathrm{c}}(0,09)$ & $0,714^{\mathrm{a}}(0,43)$ \\
\hline QtC-D & $6,12^{\mathrm{b}}(0,34)$ & $0,06^{\mathrm{d}}(8,68)$ & $0,704^{\mathrm{ab}}(0,28)$ \\
\hline QtC-E & $6,01^{\mathrm{c}}(0,69)$ & $0,08^{\mathrm{c}}(0,04)$ & $0,706^{\mathrm{ab}}(0,49)$ \\
\hline
\end{tabular}

*[A a E] Diferentes marcas comerciais de queijos de coalho comercializados em cidades do Ceará; $\left({ }^{1}\right)$ Média de três repetições \pm coeficiente de variação em \%. a, b, c, d Letras minúsculas sobrescritas distintas na mesma coluna indicam diferença significativa $(p<0,05)$ entre as marcas de queijos de coalho pelo teste de Tukey.

Fonte: Autores. Limoeiro do Norte, Ceará, Brasil (2020).

Os valores de $\mathrm{pH}$ variaram entre 5,30 a 6,29 (Tabela 1), a amostra de QtC-A se destacou por apresentar o menor valor de $\mathrm{pH}(5,30)$ e, consequentemente, o maior valor de acidez titulável; já a amostra de QtC-B apresentou o menor valor de $\mathrm{pH}$ entre todos os queijos avaliados. Pode-se observar que a amostra de QtC-B apresentou valor de pH relativamente próximo ao 
pH do leite bovino $(6,8$ a 6,6) (Dias \& Antes, 2014), o que significa que não houve ou, quase não houve uma fermentação láctea, já que a coagulação enzimática, por si só (sem adição de culturas) pode chegar a até $\mathrm{pH} \approx 6$ no queijo.

A variação nos resultados pode estar associada às condições de produção e comercialização dos queijos, como por exemplo, variação de temperatura que pode afetar o $\mathrm{pH}$ do produto, podendo provocar alterações de composição e das características sensoriais e físicas do mesmo. Assim, o pH modifica as características de textura, atividade microbiana e condição de maturação do queijo. Além disso, quando elevado pode favorecer o crescimento microbiano, e quando muito baixo pode favorecer ou facilitar a deterioração por microrganismos (Shabbir, Huma \& Javed, 2019; Sousa et al., 2014).

$\mathrm{O}$ pH é considerado uma determinação mais importante para caracterizar queijos, devido à influência na textura, na atividade microbiana e na maturação, já que ocorrem reações químicas que são catalisadas por enzimas provenientes do coalho e da microbiota, que dependem do $\mathrm{pH}$ (Sousa et al., 2014). Freitas et al. (2013) encontraram valores de pH que variaram entre 4,8 a 5,6, na avaliação da qualidade de queijo coalho produzidos no estado da Paraíba, dados esses que divergem um pouco dos encontrados para a maioria das amostras do presente estudo (QtC-B, C, D e E).

As amostras de QtC analisadas podem ser consideradas como um alimento de baixa acidez, de acordo com Franco \& Landgraf, (2005), que classificam alimentos de baixa acidez como aqueles que apresentam pH > 4,50. As amostras de QtC do presente trabalho apresentaram pH entre 5,30 a 6,29, respectivamente (Tabela 1), desta forma, as amostras de QtC são mais sujeitas à multiplicação por microrganismos deteriorantes e patógenos e, ainda mais sujeitos devido à elevada atividade de água das amostras de queijo.

Para o parâmetro acidez titulável (mg de ácido lático.g $\mathrm{g}^{-1}$ ) as amostras de QtC apresentaram grande variação, variando entre 0,06 a $0,28 \mathrm{mg}$ de ácido lático.g $\mathrm{g}^{-1}$. A acidez titulável é oriunda da degradação da lactose em ácido lático por bactérias específicas, e tem relação direta com o $\mathrm{pH}$, desta forma valores muito elevados de acidez são associados à baixos $\mathrm{pH}$, que podem facilitar a contaminação microbiana. Sousa et al. (2014), avaliaram os aspectos físico-químicos e microbiológicos de queijo de coalho comercializado em estados do Nordeste e obtiveram valores de 0,12 a 1,01\%, valores esses discrepantes para a maioria das amostras analisadas no presente estudo (QtC-C, D e E).

Sousa et al. (2014) enfatizam também que a acidez decorrente da produção de ácido lático, tem influência direta no pH e na expulsão de soro da massa durante a fabricação e na fase inicial da cura, sendo um parâmetro muito importante para manter a qualidade do produto, embora não haja na legislação valores de referência para as análises de atividade de água, acidez e pH do queijo de coalho, os valores encontrados neste trabalho foram semelhantes a alguns trabalhos citados da literatura.

Os valores da atividade de água $\left(\mathrm{A}_{\mathrm{w}}\right)$ das amostras de QtC (Tabela 1) variaram significativamente entre si, de maneira geral, foi observado que existiu semelhança significativa $(p>0,05)$ entre as amostras de QtC-D e E e, essas com as demais amostras de QtC-A, B e C. Entretanto, também se observou diferença significava $(p<0,05)$, entre a amostra de QtC-A e as amostras de QtC-B e C. Os dados encontrados neste estudo revelam que 100\% das amostras analisadas apresentaram valores acima de 0,600 para a atividade da água, o que indica a necessidade de uma atenção maior na preservação da qualidade dos queijos.

Valores muito altos de atividade de água tornam os queijos mais susceptíveis à proliferação microbiana, quando não se tem os cuidados de temperatura controlados de armazenamento durante a comercialização, ou se durante o transporte ocorre alguma oscilação na mesma. Sousa et al. (2014) encontraram valores que variaram entre 0,890 e 0,960 nas amostras de queijo de coalho analisadas, valores esses superiores aos encontrados no presente trabalho. Freitas et al. (2013) obtiveram valores variando entre 0,970 a 0,970, estando também acima dos obtidos neste estudo.

Na Tabela 2 podem ser observados os valores de $L^{*}, a^{*}$ e $b^{*}$. Com relação aos resultados obtidos para a coordenada colorimétrica $L^{*}$. Observou-se que todas as marcas de QtC não diferiram estatisticamente entre si $(p>0,05)$. O valor $L^{*}$ nos 
fornece a luminosidade, variando do branco $(\mathrm{L}=100)$ ao preto $(\mathrm{L}=0)$. Diante do exposto, pode-se afirmar que todas as marcas de QtC apresentaram cor com uma tendência ao branco.

Tabela 2. Análise de cor [média $(\mathrm{CV} \%)]^{1}$ dos queijos de coalho comercializados em cidades do Ceará.

\begin{tabular}{lccc}
\hline \multirow{2}{*}{ Marcas de QtC* } & \multicolumn{3}{c}{ Parâmetros de Cor } \\
\cline { 2 - 4 } & $\boldsymbol{L}^{*}$ & $\boldsymbol{a}^{*}$ & $\boldsymbol{b}^{*}$ \\
\hline QtC-A & $84,83^{\mathrm{a}}(0,55)$ & $3,78^{\mathrm{bc}}(2,68)$ & $24,81^{\mathrm{b}}(2,66)$ \\
QtC-B & $86,08^{\mathrm{a}}(0,28)$ & $3,11^{\mathrm{c}}(9,17)$ & $21,98^{\mathrm{c}}(3,13)$ \\
QtC-C & $86,32^{\mathrm{a}}(0,61)$ & $3,62^{\mathrm{bc}}(10,94)$ & $24,10^{\mathrm{b}}(2,08)$ \\
QtC-D & $87,17^{\mathrm{a}}(1,49)$ & $4,38^{\mathrm{b}}(8,15)$ & $24,64^{\mathrm{b}}(2,64)$ \\
QtC-E $^{\mathrm{b}}$ & $86,47^{\mathrm{a}}(0,85)$ & $6,07^{\mathrm{a}}(2,10)$ & $31,21^{\mathrm{a}}(1,59)$ \\
\hline
\end{tabular}

* [A a E] Diferentes marcas comerciais de queijos tipo Coalho comercializados em cidades do Ceará; $\left({ }^{1}\right)$ Média de três repetições \pm coeficiente de variação em $\% ; L^{*}=$ luminosidade; $a^{*}=$ Quando - grau de esverdiamento e, quando + grau de vermelhidão; $b^{*}=$ Quando grau de azulidade e, quando + , grau de amarelado. ${ }^{\mathrm{a},} \mathrm{b}, \mathrm{c}$ Letras minúsculas sobrescritas distintas na mesma coluna indicam diferença significativa $(p<0,05)$ entre as marcas de queijos de coalho pelo teste de Tukey.

Fonte: Autores. Limoeiro do Norte, Ceará, Brasil (2020).

Não se verificou o mesmo comportamento estatístico do parâmetro $L^{*}$ [luminosidade] para os parâmetros $a^{*}$ [coloração no intervalo do vermelho $\left(+\mathrm{a}^{*}\right)$ ao verde $\left.\left(-\mathrm{a}^{*}\right)\right]$ e $b^{*}$ [coloração no intervalo do amarelo $\left(+b^{*}\right)$ ao azul $\left(-b^{*}\right)$ ], que apresentaram diferença significativa $(\mathrm{p}<0,05)$ entre as marcas de QtC. Para a coordenada colorimétrica $a^{*}$, a marca de QtC-E apresentou diferença significativa ao nível de significância de 5\% entre todas as demais marcas de QtC. Ainda houve semelhança significativa entre as marcas de QtC- $[(\mathrm{A}, \mathrm{B}$ e C) e, (A, C e D)]. O mesmo comportamento foi observado para a coordenada colorimétrica $b^{*}$, a qual, a marca de QtC-E apresentou diferença significativa $(\mathrm{p}<0,05)$ entre todas as demais marcas de QtC. Entretanto, a marca de QtC-B também diferiu significativamente ( $<<0,05)$ de todas as demais marcas de QtC. Houve semelhança significativa entre as marcas de QtC-A, C e D para a coordenada colorimétrica $b^{*}$. Sendo a intensidade das cores vermelho e amarelo mensuradas pelos valores positivos, quanto maior os seus valores, mais vermelho e amarelo será a coloração das marcas de QtC. Por isso, verificou-se que todas as marcas de QtC tenderam a cor vermelha e amarela para os parâmetros $a_{(+)}^{*}$ e $b_{(+)}^{*}$, respectivamente.

A cor é uma característica importante da aparência, pois é percebida logo no primeiro contato do consumidor com o produto e pode fornecer informações sobre o seu processamento e, também pode demonstrar muito de sua qualidade. De acordo com a Instrução Normativa $n^{\circ}$ 30, de 26 de Junho de 2001 (Brasil, 2001), o queijo de coalho deve ser branco amarelado e uniforme, que se evidencia de acordo com os valores encontrados nas análises de cor, visto que os dados de $L^{*}$ (Luminosidade) variaram de 84,83 a 87,17, ou seja, quanto mais próximo de 100 mais claro o objeto analisado. Segundo a escala de análise, bem como os dados encontrados para $b_{(+)}^{*}$, quando resultados positivos tendem à coloração amarela, variaram de 21,98 a 31,21, estando de acordo com a Instrução Normativa nº 30, de 26 de Junho de 2001 (Brasil, 2001).

Tal parâmetro contribui para uma melhor caracterização do produto analisado e evidencia a qualidade do mesmo, mostrando uma padronização no processo de fabricação, o que contribui também para uma confiança no ato da compra pelos consumidores, visto que as caraterísticas sensórias dos alimentos são os primeiros impactos diante da escolha pelos consumidores.

Na Tabela 3, encontra-se a composição centesimal das diferentes marcas de Queijos de Coalho (QtC). Quanto ao teor de umidade, estatísticamente a marca de QtC-B apresentou diferença significativa $(\mathrm{p}<0,05)$ entre todas as demais marcas de QtC. As marcas de QtC-C, D e E) não diferiram significativamente entre si ( $p>0,05)$, e simultaneamente foi observado uma diferença significativa $(\mathrm{p}<0,05)$ entre a marca de $\mathrm{QtC}-\mathrm{A}$ e a marca $(\mathrm{D})$ de $\mathrm{QtC}$ e apresentou uma semelhança significativa entre as marcas de QtC-A, C e E. 
Tabela 3. Composição centesimal [média $(\mathrm{CV} \%)]^{1}$ dos queijos tipo de coalho comercializados em cidades do Ceará.

\begin{tabular}{|c|c|c|c|c|c|c|}
\hline \multirow{2}{*}{$\begin{array}{l}\text { Marcas } \\
\text { de QtC* }\end{array}$} & \multicolumn{5}{|c|}{ Composição centesimal $\left(\%, \mathrm{~g} .100 \mathrm{~g}^{-1}\right)^{\#}$} & \multirow{2}{*}{$\mathrm{VCT}^{2}$} \\
\hline & Umidade & Cinzas & Proteína & Lipídios & $\mathbf{C T}^{\mathbf{1}}$ & \\
\hline QtC-A & $44,48^{\mathrm{b}}(0,90)$ & $4,12^{\mathrm{d}}(0,37)$ & $21,78^{\mathrm{a}}(1,72)$ & $26,27^{\text {bc }}(3,89)$ & $2,79^{\mathrm{b}}(18,56)$ & $339,07^{\mathrm{b}}(0,04)$ \\
\hline QtC-B & $47,52^{\text {a }}(0,58)$ & $4,24^{\mathrm{d}}(1,53)$ & $21,99^{\text {a }}(0,70)$ & $24,17^{\mathrm{c}}(3,16)$ & $2,44^{\mathrm{b}}(3,78)$ & $313,37^{\mathrm{c}}(0,35)$ \\
\hline QtC-C & $43,10^{\mathrm{bc}}(0,11)$ & $4,40^{\mathrm{c}}(1,13)$ & $20,96^{\mathrm{b}}(0,05)$ & $29,17^{\text {a }}(0,99)$ & $2,37^{\mathrm{b}}(12,16)$ & $355,84^{\mathrm{a}}(0,42)$ \\
\hline QtC-D & $41,75^{\mathrm{c}}(0,56)$ & $4,63^{\mathrm{b}}(1,32)$ & $21,99^{\text {a }}(0,76)$ & $27,60^{\mathrm{ab}}(4,41)$ & $4,77^{\mathrm{a}}(1,77)$ & $348,75^{\mathrm{ab}}(0,43)$ \\
\hline QtC-E & $42,80^{\mathrm{bc}}(5,04)$ & $4,81^{\mathrm{a}}(0,89)$ & $22,17^{\text {a }}(0,02)$ & $28,08^{\mathrm{ab}}(2,24)$ & $2,74^{\mathrm{b}}(13,73)$ & $355,00^{\mathrm{a}}(1,76)$ \\
\hline
\end{tabular}

* [A a E] Diferentes marcas comerciais de queijos tipo Coalho comercializados em cidades do Ceará; $\left({ }^{1}\right)$ Média de três repetições e coeficiente de variação em \%; $\left(^{\#}\right)$ Valores expressos em base úmida, $\%=\mathrm{g} .100 \mathrm{~g}^{-1}$; $\left.{ }^{1}\right)$ Carboidratos por diferença. $\left({ }^{2}\right)$ Valor Calórico Total, expresso em kcal.100 $\mathrm{g}^{-1}$. a, b, c Letras minúsculas sobrescritas distintas na mesma coluna indicam diferença significativa $(p<0,05)$ entre as marcas de queijos de coalho pelo teste de Tukey.

Fonte: Autores. Limoeiro do Norte, Ceará, Brasil (2020).

Apesar dos teores de umidades terem variados de 41,75 a 47,52\%, de acordo com o Regulamento Técnico de Identidade e Qualidade de queijos (Brasil, 1996), os QtC do presente trabalho podem ser classificados como queijos de alta umidade $(46,0$ a $54,9 \%)$.

Vidal (2011) obteve valores entre 32,97 e 39,77\% de umidade, para queijos de coalho, que obteve valores abaixo do encontrado no presente estudo, bem como Sousa et al. (2014) que encontraram teores de umidade que variaram de 14,38 a 24,08\%. Este último apresentou-se também fora do limite estabelecido pela legislação, atribui-se esses valores a falta de padronização na elaboração do queijo, formação e manuseio da coalhada afetam a sua habilidade de reter a umidade, influenciando nessa variação do teor de umidade.

Sousa et al. (2014) obtiveram dados de umidade para queijos de coalho numa variação de 14,38 a 24,08\% para os produtos com inspeção, e de 15 a 29,38\% para os de fabricação artesanal, caracterizando, assim, os queijos não estavam em conformidade com a legislação (36 a 54,9\%).

Com relação aos teores encontrados na determinação de cinzas, observou-se semelhança significativa $(p>0,05)$ entre as marcas de QtC-A e B, que diferiram estatisticamente das demais marcas de QtC. As marcas de QtC (C, D e E) apresentaram diferença significativa $(p<0,05)$ entre si em todas as demais marcas de QtC (Tabela 3). Verificou-se que a marca de QtC-E apresentou valor estatisticamente superior em minerais $\left(4,81 \mathrm{~g} .100 \mathrm{~g}^{-1}\right)$, quando comparado às demais marcas de QtC. Valores semelhantes foram encontrados por Gomes, Medeiros \& Silva (2012), em queijos de coalho, com 4,47 e 4,88\% de cinzas. Já Silva et al. (2020) encontraram valores de cinzas de 4,07 a 5,36 para queijos de coalho, dados considerados um pouco superiores aos encontrados na presente pesquisa.

Quanto aos teores de proteína (Tabela 3), foi observado valor estatisticamente inferior para a marca de QtC-C, que apresentou o menor valor de proteínas entre as marcas de QtC do presente trabalho. As demais marcas de QtC foram estatisticamente $(p>0,05)$ semelhantes entre si.

Silva et al. (2010) encontraram valores de 26,93 e 29,63\% para proteínas, dados acima dos resultados das amostras de queijo de coalho analisadas na presente pesquisa, tais autores relatam que as características sensoriais dos queijos, bem como sua composição, são afetadas muitas vezes pela falta de padronização no processo de fabricação dos mesmos, como a utilização de diferentes tipos de leites (cru, desnatado ou pasteurizado), estes podem apresentar-se como substrato para desenvolvimento de vários microrganismos, capazes de afetar as características do mesmo ou, até mesmo, comprometer sua qualidade (Sola et al., 2020).

Os teores de lipídios das marcas de QtC são mostrados na Tabela 3, os mesmos mostraram diferença estatística significativa $(p<0,05)$ somente entre as marcas de QtC-[(B e C) e, (C e A)]. Já as amostras das marcas de QtC-[(A e B), (A, D e E) e, $(C, D$ e E)] apresentaram semelhança significativa $(p>0,05)$ entre si. 
A legislação brasileira, Brasil (2001) estabelece um teor de gordura nos sólidos totais variável entre 35 a $60 \%$. As marcas de QtC do presente trabalho estão em acordo com a legislação brasileira, apresentando em base seca (sólidos totais) valores de gordura (coeficiente de variação em \%) de: $\mathrm{QtC}-\mathrm{A}=47,31(3,89), \mathrm{B}=46,05(3,16), \mathrm{C}=51,26(0,99), \mathrm{D}=47,38$ $(4,41)$ e $\mathrm{E}=49,10$ (2,24). A legislação Brasileira (Brasil, 1996) classifica os queijos por meio do seu conteúdo de gordura, em extra gordo ou duplo creme: quando contenham o mínimo de 60\%; gordos: quando contém entre 45,0 e 59,9\%; semigordo: entre 25,0 e 44,9\%; magros: entre 10,0 e 24,9\% e, desnatados: quando contém menos de 10,0\%. Os QtC do presente trabalho obtiveram teor de gordura entre 24,17 a 29,17, sendo os QtC-A, C, D e E classificados com a denominação de "queijo semigordo" e, o QtC-B, com a denominação de "queijo magro".

Gomes, Medeiros \& Silva (2012), encontraram resultados semelhantes ao do presente estudo com teores de 23,30 e 27,30\% para lipídios (resultados expressos em base úmida) nos queijos de coalho, estando também de acordo com a legislação vigente para esse produto. Silva et al. (2010) encontraram para queijo de coalho teores de 36,59 a 48,16\% de lipídio [resultados expressos em base seca (sólidos totais)], dentro do esperado pela legislação. Entretanto, tais resultados podem afirmar que a matéria-prima, o leite utilizado no processamento dos queijos diverge bastante quanto à composição e, consequentemente os constituintes centesimais dos queijos, principalmente gordura e proteína serão divergentes entre os mesmos. Miloradovic et al. (2015), que avaliaram diferentes tipos de queijos a base de leite de cabra e, constataram que o tipo de leite utilizado influenciou significativamente no teor de gordura no produto final.

Os teores de carboidratos totais das marcas de QtC estão apresentados na Tabela 3, sendo que estatísticamente a marca de QtC-D diferiu significativamente $(p<0,05)$ das demais marcas de QtC, destacando-se das demais por apresentar maior percentual de carboidratos. O valor calórico total em Kcal.100 g g $^{-1}$ (Tabela 3) mostrou que as marcas de QtC-[(A e D) e $(\mathrm{C}, \mathrm{D}$ e E)] não diferiam estatísticamente entre si $(p>0,05)$. Entretanto, a marca de QtC-C diferiu significativamente a nível de 5\% de significância com as demais marcas de QtC. Ainda, existiu diferença significativa entre as marcas de QtC-A, C e E.

Os resultados encontrados de valor calórico total para as marcas de QtC foram de 313,37 a 355,8 Kcal.100 g g $^{-1} \mathrm{O}$ valor calórico total é a quantidade de energia que o organismo recebe durante a assimilação completa do alimento no processo da digestão. Silva et al. (2020) encontraram valores de 352,43 a 378,26 Kcal.100 $\mathrm{g}^{-1}$ para queijos de coalho comercializados em feiras livres de São Luis-MA, dados acima dos encontrados no presente estudo. Já Oliveira et al. (2018) encontraram valores que oscilaram de 254,88 a 339,14 Kcal.100 g-1 dados próximos dos encontrados na pesquisa em questão.

A discrepância entre os valores encontrados na presente pesquisa e os valores relatados nos trabalhos evidenciam que a composição do queijo é caracterizada segundo a matéria-prima utilizada no seu processo de elaboração. Miloradovic et al. (2015) citam que o tratamento térmico também influencia diretamente na composição dos queijos produzidos e em suas características sensoriais como a cor. Além disso, observaram que os queijos produzidos com leite de cabra apresentaram dados de cor bem discrepantes; já os queijos elaborados com leite de vaca não apresentaram grandes variações no parâmetro cor, de acordo com os dados encontrados na presente pesquisa (Tabela 2).

\section{Conclusão}

Conclui-se que as marcas de queijo de coalho comercializados em cidades do Ceará, apresentaram-se dentro dos padrões de composição centesimal, como estabelecido pela legislação brasileira vigente. Os parâmetros físico-químicos dos queijos apresentaram menores variações entre as amostras e dentro dos valores médios encontrados na literatura para queijo de coalho. Todas as marcas de queijo de Coalho apresentaram cor com uma tendência ao amarelado, com exceção das amostras C que ficaram brancas.

Entretanto, as variações de composição centesimal encontradas nas amostras podem ser justificadas por se tratarem principalmente de marcas diferentes de queijo comercializados em cidades diferentes do Ceará e, as variações podem ser 
principalmente associadas à matéria-prima para produção dos queijos, como também associadas às condições de produção, variação de temperatura do processamento, armazenamento e comercialização dos queijos. A atual pesquisa aponta para a necessidade de ampliação dos parâmetros de controle de qualidade presentes na legislação que estabelece padrões de identidade e qualidade referentes ao queijo de coalho, com a finalidade de garantir maior controle de fabricação dos queijos de coalhos mais seguros pelas diferentes unidades produtores.

Sugere-se para trabalhos futuros pesquisas sobre a qualidade microbiológica dos queijos de coalho, bem como uma análise descritiva de todo o percurso que esse produto faz até ao consumidor.

\section{Agradecimentos}

Os autores agradecem a Coordenação de Aperfeiçoamento de Pessoal de Nível Superior (CAPES), bem como a Fundação Cearense de Apoio ao Desenvolvimento Científico e Tecnológico (FUNCAP) pela bolsa de estudos cedida aos alunos participantes do trabalho (Mestrado/IFCE).

\section{Referências}

Atwater, W. O. (1910). Principles of nutrition and nutritive value of food. (Farmers' Bulletin 142). Washington: Government Printing Office, US Department of Agriculture. 141. https://ia800301.us.archive.org/15/items/principlesofnutr00atwa/principlesofnutr00atwa.pdf.

Brasil. Ministério da Agricultura e do Abastecimento Secretaria de Defesa Agropecuária. Instrução Normativa N 30 , de 26 de Junho de 2001 . Aprovar os Regulamentos Técnicos de Identidade e Qualidade de Manteiga da Terra ou Manteiga de Garrafa; Queijo de Coalho e Queijo de Manteiga, conforme consta dos Anexos desta Instrução Normativa.

Brasil. Regulamentos Técnicos de Identidade e Qualidade dos Produtos Lácteos. In P. e. A. Ministro de Estado da Agricultura (Ed.), Portaria n 146 de 07 de março de 1996 Brasília: Ministério da Agricultura Secretaria de Defesa Agropecuária. D.O.U. — Diário Oficial da União, de 11 de março de 1996. Ahttp://www.dourados.ms.gov.br/wp-content/uploads/2016/05/RTIQ-Leite-Completo-PORTARIA-146_96-ok.pdf.

Dias, J. A., \& Antes, F. G. (2014). Qualidade físico-química, higiênico sanitária e composicional do leite cru: Indicadores e aplicações práticas da Instrução Normativa (62a ed.), 1, Documento 158, EMBRAPA, 19 p.

Ferreiro, J. L., \& Rodríguez-Otero. (2018). Evolução e distribuição de fosfolipídios em queijo e soro de leite durante a fabricação de queijo fresco a partir de leite de vaca. International Journal of Dairy Technology, 71 (3) (2018), 820 - 823. https://doi.org/10.1111/1471-0307.12499.

Franco, B. D. G. M., \& Landgraf, M. (2005). Microbiologia dos alimentos, Atheneu, 196p.

Freitas, W. C., Travassos A. E. R., \& Maciel, J. F. (2013). Avaliação microbiológica e físico-química de leite cru e queijo de coalho produzidos no estado da Paraíba. Revista Brasileira de Produtos Agroindustriais. 15(1), 35-42.

Garcia-Gómez, B., Vázquez-Odériz, M. L., Muñoz-Ferreiro, N., Romero-Rodríguez, M. Á., \& Vázquez, M. (2019). Interaction between rennet source and transglutaminase in white fresh cheese production: effect on physicochemical and textural properties: Effect on physicochemical and textural properties. $\mathbf{L} \boldsymbol{w}$, 113, 108279-108287, http://dx.doi.org/10.1016/j.lwt.2019.108279.

Gomes, R. A., Medeiros, U. K. L de., \& Silva, F. A. P da. (2012). Caracterização físico-química dos Queijos de Coalho artesanal e industrial comercializados na cidade de Currais Novos/RN. CONNEPI.

Instituto Adolo Lutz. (2008). Métodos físico-químicos para análise de alimentos. (4a ed.), IMESP.

Liao, H., Zhong, K., Hu, X., \& Liao, X. (2019). Effect of high pressure carbon dioxide on alkaline phosphatase activity and quality characteristics of raw bovine milk. Innovative Food Science \& Emerging Technologies, 52, 457-462, http://dx.doi.org/10.1016/j.ifset.2019.02.005.

Miloradovic, Z. N., Kljajevic, N. V., Jovanovic, S. T., Vucic, T. R., \& Macej, O. D. (2015). The effect of heat treatment and skimming on precipitate formation in caprine and bovine milks. Journal of Dairy Research, 82, 22-28.

Oliveira, E. N. A., Santos, D. C., Almeida, F. L. C., Feitosa, B. F., \& Feitosa, R. M. (2018). Caracterização de queijos artesanais comercializados em municípios do Ceará. Revista Exacta, 11(2), 55-62.

Pereira, A. S., Shitsuka, D. M., Parreira, F. J., \& Shitsuka, R. (2018). Metodologia da pesquisa científica. UFSM, 119p.

Reis, P. J. M. \& Malcata, F. X. (2011). Ripening-related changes in Serra da Estrela cheese: A stereological study. J. Dairy Sci. 94, 1223-1238. $10.3168 /$ jds.2010-3416.

Rosseto, M., Batistella, V. M. C., \& Veiga, R. L. (2020). Análise de perigos e pontos críticos de controle: um estudo de caso em uma propriedade leiteira do Município de Sertão, Rio Grande do Sul, Brasil. Research, Society and Development, 9(8), e69985136. http://dx.doi.org/10.33448/rsd-v9i8.5136.

R Core Team. (2018). R: A language and environment for statistical computing. R Foundation for Statistical Computing. 
Research, Society and Development, v. 10, n. 2, e46710212573, 2021

(CC BY 4.0) | ISSN 2525-3409 | DOI: http://dx.doi.org/10.33448/rsd-v10i2.12573

Santiago-López, L., Aguilar-Toalá, J. E., Hernández-Mendoza, A., Vallejo-Cordoba, B., Liceaga, A. M., \& González-Córdova, A. F. (2018). Invited review: bioactive compounds produced during cheese ripening and health effects associated with aged cheese consumption.: Bioactive compounds produced during cheese ripening and health effects associated with aged cheese consumption. Journal Of Dairy Science, 101(5), 3742-3757. American Dairy Science Association. http://dx.doi.org/10.3168/jds.2017-13465.

Selin, A., Demet, E., Çağım, A. Ç., Hasan, V., Asliye, K., \& Mehmet, K. (2018). Efeito do coalho de Oryctolagus cuniculus (coelho) na textura, reologia e propriedades sensoriais do queijo branco. Ciência e Nutrição Alimentar, 6 (4), 1100 - 1108. https://doi.org/10.1002/fsn3.649.

Shabbir, U., Huma, N., \& Javed, A. (2019). Composição e propriedades de textura de queijo de leite de cabra preparado usando dahi (iogurte) como iniciador. Revista Brasileira de Tecnologia de Alimentos, 22, 1-7. https://doi.org/10.1590/1981-6723.28918.

Silva, M. C. D., Ramos, A. C. S., Moreno, I., \& Moraes, J. O. (2010). Influência dos procedimentos de fabricação nas características físico-químicas, sensoriais e microbiológicas de queijo de coalho. Revista Instituto Adolfo Lutz. 69(2):214-21.

Silva, B. P. P., Oliveira, R. W. S., Sousa, I. B., Gomes, P. R. B., Santos, S de J. L., Louzeiro, H. C., Melo, A. V., Reis, J. B., Lima, H. S., Silva, E. F., Fontenele, M. A., Paula, M do. L., \& Mouchrek Filho, V. E. (2020). Nutritional composition of coalho cheese sold at free fairs in São Luis - MA, Braz. J. of Develop, Curitiba, 6(6), 34043-34053..10.34117/bjdv6n6-088.

Sola, M. C., Feistel, J. C., Freitas, F. A., Fioravanti, M. C. S., Soares Juliano, R., \& Rezende, C. S. M e. (2020). Perfil microbiológico do leite das raças bovinas Curraleiro Pé-Duro e Pantaneiro. Research, Society and Development, 9(11), e45691110194. http://dx.doi.org/10.33448/rsd-v9i11.10194.

Sousa, A. Z. B., Abrantes, M. R., Sakamoto, S. M., Silva, J. B. A., Lima, P. de O., Lima, R. N., Rocha, M. de O. C., \& Passos, Y. D. B. (2014). Aspectos físico-químicos e microbiológicos do queijo tipo coalho comercializado em estados do nordeste do Brasil. Arq. Inst. Biol. 81(1), 30-35.

Vidal, R. H. L. (2011). Diagnóstico regional do processo de queijo coalho comercializado em Natal/RN. (Dissertação). Natal (RN): Universidade Federal do Rio Grande do Norte; 2011. 